\title{
NOTA
}

\section{POTENCIAL DE PRODUÇÃO DE SEMENTES DE CULTIVARES E CLONES DE ABACAXI VISANDO AO MELHORAMENTO GENÉTICO $\left(^{1}\right)$}

\author{
ADEMAR SPIRONELLO $\left({ }^{2,5}\right)$, JOSÉ ALFREDO USBERTI FILHO $\left({ }^{3,5}\right)$, \\ WALTER JOSÉ SIQUEIRA $\left({ }^{3,5}\right)$, JOAQUIM TEÓFILO SOBRINHO $\left({ }^{4,5}\right)$, \\ MARLI HARRIS $\left({ }^{6}\right)$ e ANA CLÁUDIA DE CARVALHO BADAN $\left({ }^{6}\right)$
}

\begin{abstract}
RESUMO
Foram realizados, por três anos consecutivos, cruzamentos dirigidos e ao acaso entre 18 cultivares e clones de abacaxi, Ananas comosus (L.) Merrill, visando obter progênies segregantes quanto à resistência à fusariose e para caracteres de planta e de fruto, objetivando a seleção futura de clones superiores. Os materiais genéticos em estudo foram avaliados quanto ao potencial de produção de sementes, em dois sistemas de polinização, para fornecer subsídios ao melhorista no direcionamento das hibridaçôes. Observou-se elevada variação na quantidade de sementes produzidas nos cultivares e clones testados. Nos dois tipos de cruzamento, sobressaíram-se Rioja, Amarelo-de-uaupés e Perolera; nos cruzamentos ao acaso, também Roxo-de-tefé e Boituva, e, nos dirigidos, Natal Queen. Houve alta correlação $\left(r=0,82^{* *}\right)$ entre esses dois tipos de cruzamento.
\end{abstract}

Termos de indexação: abacaxi, Ananas comosus (L.) Merrill, melhoramento genético, cruzamentos, produção de sementes.

(1) Recebido para publicação em 21 de março e aceito em 2 de junho de 1994. Pesquisa parcialmente financiada pela Fundação de Amparo à Pesquisa do Estado de São Paulo (FAPESP).

( ${ }^{2}$ ) Seção de Fruticultura Tropical, Instituto Agronômico (IAC), Caixa Postal 28, 13001-970 Campinas (SP).

$\left(^{3}\right)$ Seção de Genética (IAC).

(4) Centro de Citricultura "Sylvio Moreira" (IAC).

(5) Com bolsa de pesquisa do CNPq.

(6) Com bolsa de aperfeiçoamento do $\mathrm{CNPq}$. 


\title{
ABSTRACT \\ SEED YIELD POTENTIAL OF CULTIVARS AND CLONES OF PINEAPPLE IN ORDER TO SUPPORT PLANT BREEDING
}

\begin{abstract}
Open-pollinated and artificial crosses have been carried out throughout three years among 18 cultivars and clones of pineapple, Ananas comosus (L.) Merrill, aiming at the future selection of new hybrids with desirable agronomic and fruit quality traits, in segregating progenies. The genetic materials have been evaluated as to their seed yield potentials in order to provide basic information for the choice of parentals to be used in future crosses. The amount of viable seeds produced in different crosses has been higly variable. Cultivar Perolera and clones Amarelo-deuaupés, Roxo-de-tefé, Rioja, and Boituva have been the best seed producers in open-pollinated crosses whereas cultivar Perolera and clones Amarelo-de-uaupés, Natal Queen, and Rioja outyielded the others in artificial crosses. A significant correlation $\left(r=0.82^{* *}\right)$ was observed between the seed yields obtained in the two pollination systems used.
\end{abstract}

Index terms: pineapple, Ananas comosus (L.) Merrill, plant breeding, crosses, seed production.

O Instituto Agronômico de Campinas (IAC) conta, atualmente, com um banco de germoplasma de abacaxi constituído de 50 cultivares comestíveis, Ananas comosus (L.) Merrill, 13 espécies selvagens e cinco variedades botânicas de Pseudananas ( $\mathrm{sem}$ coroa).

A fusariose, causada pelo fungo Fusarium moniliforme Sheld. var. subglutinans, ć, atualmente, a principal doença limitante à produção de frutos. Os cultivares comerciais em cultivo em escala (Smooth Cayenne e Pérola) são altamente suscetíveis ao patógeno. O método de controle mais racional e eficiente da fusariose é o emprego de cultivares resistentes, obtidos mediante o melhoramento genético.

Iniciou-se, no IAC, um programa de melhoramento genético de abacaxi visando à resistência à fusariose, por meio de hibridações ao acaso e artificialmente dirigidas, entre diferentes materiais genéticos do banco de germoplasma. Tornou-se, então, necessário o conhecimento do potencial de produção de sementes dos cultivares e clones disponíveis.

Os cruzamentos foram concentrados em cultivares e clones com comprovada resistência à fusariose, com folhas inermes e folhas com espinhos, e em outros que, embora suscetíveis à doença, são portadores de características agronômicas ou tecnológicas desejáveis (Smooth Cayenne, Pérola, Natal Queen, Boituva, Guiana e San Ramón).

\section{Material e Métodos}

Os cultivares e clones de abacaxi utilizados neste trabalho e algumas de suas principais características encontram-se no quadro 1.

O banco ativo de germoplasma de abacaxi do IAC, localizado em pequena área no Centro de Citricultura (Cordeirópolis, SP), é constituído de linhas de cultivares e clones de comprimento e disposição variáveis, com espaçamento de $0,40 \mathrm{~m}$ entre plantas (dentro da linha) e de $1,20 \mathrm{~m}$ entre linhas. Utilizaram-se, também, plantas de experimentos com variedades desse local e da Estação Experimental de Votuporanga.

Durante os anos agrícolas de 1991/92, 1992/93 e 1993/94, à medida que os indivíduos dos cultivares e clones escolhidos entravam em estádio de emissão de flores, as inflorescências eram cuidadosamente protegidas com sacos de papel impermeável. No início da antese contínua, que ocorre da base para o ápice da inflorescência (durante duas a três semanas), as folhas abertas foram polinizadas com pólen coletado em outros clones e cultivares. 
Quadro 1. Características principais de cultivres e clones utilizados neste trabalho

\begin{tabular}{|c|c|c|c|c|c|c|}
\hline \multirow{2}{*}{ Clone (cl) e Cultivar (cv) } & \multirow{2}{*}{$\begin{array}{l}\text { Resistência } \\
\text { à fusariose }\end{array}$} & \multirow{2}{*}{$\begin{array}{l}\text { Espinhosidade } \\
\text { foliar }\end{array}$} & \multirow{2}{*}{$\begin{array}{l}\text { Tamanho } \\
\text { do fruto }\end{array}$} & \multicolumn{3}{|c|}{ Polpa } \\
\hline & & & & Cor & Doçura & Acidez \\
\hline Perolera (cv) & Elevada & Ausente & Grande & Amarela & Elevada & Média \\
\hline Rondon (cl) & Elevada & Ausente & Médio & Branca & Elevada & Baixa \\
\hline Manzana (cl) & Elevada & Ausente & Grande & Amarela & Média & Elevada \\
\hline Tapiracanga $(\mathrm{cl})$ & Elevada & Ausente & Médio & Branca & Elevada & Elevada \\
\hline Manauara Inerme (cl) & Elevada & Ausente & Médio & Branca & Média & Baixa \\
\hline Roxo Inerme (cl) & Elevada & Ausente & Médio & Amarela & Elevada & Baixa \\
\hline Piña Negra (cl) & Elevada & Ausente & Médio & Amarela & Elevada & Baixa \\
\hline Roxo-de-tefé (cl) & Elevada & Total & Médio & Amarela & Elevada & Baixa \\
\hline Amarelo-de-uaupés (cl) & Elevada & Total & Pequeno & Amarela & Elevada & Baixa \\
\hline Cabezona (cl) & Elevada & Total & Grande & Branca & Elevada & Média \\
\hline Rioja (cl) & Elevada & Total & Grande & Amarela & Média & Elevada \\
\hline Huitota (cl) & Elevada & Total & Pequeno & Branca & Elevada & Baixa \\
\hline Turi Verde $(\mathrm{cl})$ & Moderada & Total & Médio & Branca & Elevada & Elevada \\
\hline Natal Queen (cl) & Moderada & Total & Pequeno & Amarela & Elevada & Baixa \\
\hline San Ramón (cl) & Moderada & Total & Grande & Amarela & Elevada & Média \\
\hline Guiana $(\mathrm{cl})$ & Baixa & Total & Médio & Amarela & Elevada & Média \\
\hline Boituva (cv) & Baixa & Total & Grande & Amarela & Elevada & Elevada \\
\hline Pérola (cv) & Baixa & Total & Médio & Branca & Elevada & Baixa \\
\hline Smooth Cayenne (cv) & Baixa & Parcial & Grande & Amarela & Elevada & Elevada \\
\hline
\end{tabular}

Fontes: Souto, 1981; Giacomelli \& Téofilo Sobrinho, 1984; Cabral et al., 1988; Espironelo \& Teófilo Sobrinho, 1991, e observações recentes do primeiro autor do presente trabalho.

À medida que as flores das plantas-mãe foram sendo polinizadas, procedeu-se ao pincelamento das sépalas das flores com tinta esmalte para identificação posterior dos frutilhos com sementes híbridas. Ao término de cada série de polinizações diárias, realizadas das sete às dez horas, as inflorescências eram novamente ensacadas, sendo, finalmente, desprotegidas após o secamento de todas as suas flores.

Nos cruzamentos ao acaso, levaram-se em conta frutos de indivíduos de cada cultivar ou clone que se cruzaram livremente e que continham sementes. Considerou-se como semente viável aquela bem formada, de tegumento liso e compacta.
Foi realizada análise de correlação (Thomas \& Hills, 1977), baseada nos caracteres número de sementes viáveis por fruto (polinização aberta) e número de sementes viáveis por flor polinizada (cruzamentos dirigidos).

\section{Resultados e Discussão}

Os resultados obtidos de 1991 a 1994 para os cruzamentos ao acaso, envolvendo 13 cultivares e clones de abacaxi, encontram-se no quadro 2. Observa-se que, quanto à porcentagem de sementes viáveis, o clone Turi Verde revelou o menor valor $(29,4 \%)$ para a característica. No grupo interme- 
diário $(43,7$ a $68,4 \%)$, situaram-se os clones Tapiracanga, Natal Queen, Rondon, Pérola e Amarelo-de-uaupés e o cultivar Smooth Cayenne. Os demais apresentaram valores maiores para o caráter $(73,6$ a $82,9 \%$ ).

Ocorreu ampla variação para o número de sementes viáveis por fruto, possibilitando a classificação dos cultivares e clones testados em quatro categorias distintas quanto ao caráter, em polinização aberta: (a) maior que 100 (excelente): Rioja e Roxo-de-tefé; (b) de 50 a 100 (bom): Perolera, Amarelo-de-uaupés e Boituva; (c) de 25 a 50 (regular): Guiana, Rondon, Natal Queen e Manzana; (d) menos de 25 (ruim): Pérola, Smooth Cayenne, Tapiracanga e Turi Verde.

Verifica-se que, dos quatro cultivares comerciais, dois (Smooth Cayenne e Pérola) encontram-se no grupo de baixa produção de sementes, e um terceiro (Perolera) apresenta boa produção de sementes viáveis.
No quadro 3 , foram reunidas as famílias de cruzamentos dirigidos com o mesmo genitor feminino ( $(\boldsymbol{)})$. As médias obtidas em cada família indicam o potencial parental de cada cultivar quanto ao número de sementes viáveis por flor polinizada. Com a ressalva de que várias famílias de cruzamentos dirigidos possuem reduzido número de polinizadores diferentes $\left(\sigma^{\prime}\right)$, os resultados permitiram sua classificação em três categorias: (a) mais de uma semente viável por flor polinizada: Rioja, Natal Queen, Amarelo-de-uaupés e Perolera; (b) de 0,5 a 1,0: San Ramón, Pérola, Piña Negra, Huitota, Manzana, Roxo Inerme, Cayenne e Tapiracanga; e (c) os demais.

Corroborando dados obtidos (Cabot, 1987), o cultivar Perolera mostrou-se especialmente indicado para servir como fêmea em cruzamentos dirigidos, mesmo porque possui diversas características desejáveis de planta e fruto. Entre estas, podem ser citadas as folhas do tipo "piping", os frutos de forma cilíndrica, de cor intensa, e a polpa rica em ácido ascórbico. Também origina progênies

Quadro 2. Resultados para diversos caracteres relacionados à produção de sementes de abacaxi em cruzamentos ao acaso realizados em 13 cultivares/clones no período de 1991 a 1993

Cultivar/clone Número Número total de frutos colhidos de sementes

Sementes viáveis

\begin{tabular}{|c|c|c|c|c|c|}
\hline & & & $n .^{\circ}$ & $\%$ & n. $\%$ fruto \\
\hline Rioja & 4 & 1.878 & 1.556 & 82,9 & 389,0 \\
\hline Roxo-de-tefé & 11 & 2.099 & 1.732 & 82,5 & 157,5 \\
\hline Perolera & 8 & 606 & 519 & 85,6 & 64,9 \\
\hline Amarelo-de-uaupés & 9 & 813 & 556 & 68,4 & 61,8 \\
\hline Boituva & 27 & 1.820 & 1.446 & 79,5 & 53,6 \\
\hline Guiana & 18 & 777 & 620 & 79,8 & 34,4 \\
\hline Rondon & 44 & 2.398 & 1.346 & 56,1 & 30,6 \\
\hline Natal Queen & 28 & 1.579 & 798 & 50,5 & 28,5 \\
\hline Manzana & 5 & 178 & 131 & 73,6 & 26,2 \\
\hline Pérola & 24 & 908 & 517 & 56,9 & 21,5 \\
\hline Smooth Cayenne & 16 & 327 & 215 & 65,7 & 13,4 \\
\hline Tapiracanga & 14 & 389 & 170 & 43,7 & 12,1 \\
\hline Turi Verde & 31 & 1139 & 335 & 29,4 & 10,8 \\
\hline Totais & 239 & 14.911 & 9.941 & 66,7 & 41,5 \\
\hline
\end{tabular}


Quadro 3. Resultados para diversos caracteres relacionados à produção de sementes de abacaxi, em cruzamentos dirigidos, nos quais cada um dos 13 cultivares ou clones utilizados atuou como genitor feminino ( $(\mathbf{q})$

Cruzamento $\left({ }^{1}\right) \quad$ NFP $\left({ }^{2}\right) \quad$ NTS $\left({ }^{3}\right) \quad$ NSV $\left({ }^{4}\right) \quad$ PSV $\left({ }^{5}\right) \quad$ NSV/FP $\left({ }^{6}\right)$

\section{\& $\sigma^{\prime \prime}$}

RJ $x$ RT

RJ $x$ PN

NQ $x$ SC

NQ $x$ RD

AU $\times$ SC

PL $x$ RT

PL $x$ RJ

PL $x$ PN

PL $x$ PR

PL $x$ RD

PL $x$ NQ

PL $x$ SC

PL $x$ MZ

PL $x$ TP

PL $x$ BT

SR $x$ PL

SR $x$ RI

PR $x$ RJ

PR x SC

PR $\times$ RT

PR $x$ MI

PN x RJ

PN $x$ PL

$\mathrm{PN} \times \mathrm{SC}$

HT $x$ RD

HT $x$ PL

HT $x$ SC

$\mathrm{MZ} \times \mathrm{SC}$

$\mathrm{MZ} \times \mathrm{PL}$

$\begin{array}{rl}8 & 70 \\ 10 & 32 \\ 10 & 35\end{array}$

$27 \quad 49$

$44 \quad 120$

$57 \quad 301$

18

14

9

98

39

94

16

41

159

144

26

14

76

16

14

11

9

32

11

50

39

132

25

256

17

25

100

14

10

25

7

1

19

68

1

202

5
Média

Média

64

31

33

41

95

266

43

31

16

163

35

49

4

9

26

203
91,4

96,9

94,3

83,7

79,2

88,4

97,7

70,5

35,5

79,5

61,4

79,0

100,0

50,0

74,3

79,3

64,7

76,0

83,0

92,9

50,0

84,0

85,7

100,0

84,2

72,1

100,0

82,2

60,0

94,1
89,0
73,6

72,0

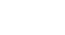

1,09

0,81

0,35

1,91

0,67

0,03

1,45

0,98

0,03

1,26

0,12

2,16

4,67

2,39

2,21

1,78

1,66

0,90

0,52

0,25

0,22

0,16

, 36

85,4

71,1
5,55

2,41

1,48

0,91

09

81

0,90

0,87

0,82
3

\begin{abstract}
60
\end{abstract}

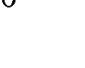


Quadro 3. Conclusão

Cruzamento $\left({ }^{1}\right) \quad$ NFP $\left({ }^{2}\right) \quad$ NTS $\left({ }^{3}\right) \quad$ NSV $\left({ }^{4}\right) \quad \operatorname{PSV}\left({ }^{5}\right) \quad$ NSV $/ F P\left({ }^{6}\right)$

\section{0}

RI $x$ SC

$\mathrm{SC} \times \mathrm{PL}$

$\mathrm{SC} \times \mathrm{RD}$

$S C \times R T$

$S C \times T V$

TP $\times$ PL

$\mathrm{TP} \times \mathrm{SC}$

RT $x$ PL

RT $x$ MI

RT $\times$ PR

RT $x$ SC

RD $\times S R$

$R D \times S C$

RD $\times$ PL

$\mathrm{RD} \times \mathrm{GN}$

RD $x$ RT

RD $\times$ NQ

RD $x \mathrm{BT}$

GN $x$ PL

GN $x$ RD

BT $x$ PL

BT $x$ SR

BT $x$ RD

$B T \times M Z$

TV $x$ PR

TV $x$ PN

TV $x$ RT

TV $\times$ PL

TV $\mathrm{x}$ MI

TV $x$ SC

CB x PL

Totais

$\begin{array}{lr}26 & 2 \\ 14 & 2 \\ 67 & 11 \\ 14 & \\ 14 & \\ 74 & 7\end{array}$

25

25

110

1

1

79

23

94

88

17

20

11

15

51

113

42

12

103

151

48

54

107

65

172

11

19

15

34

17

19

61

20

2.749

\section{7}

5

1

40

44

74

16

1

12

15

27

10

52

22

65

1

24

9

17

12

1

2

1

2.687

Média

Média

\begin{tabular}{|c|c|c|}
\hline & 0,65 & \\
\hline & 1,64 & \\
\hline & 0,36 & \\
\hline & 0,07 & \\
\hline 78,4 & 0,07 & 0,53 \\
\hline & 0,53 & \\
\hline 61,6 & 0,53 & 0,53 \\
\hline & 0,81 & \\
\hline & 0,41 & \\
\hline & 0,25 & \\
\hline 93,9 & 0,09 & 0,39 \\
\hline & 1,07 & \\
\hline & 0,59 & \\
\hline & 0,46 & \\
\hline & 0,38 & \\
\hline & 0,08 & \\
\hline & 0,07 & \\
\hline 68,1 & 0,04 & 0,38 \\
\hline & 0,56 & \\
\hline 100,0 & 0,19 & 0,37 \\
\hline & 0,43 & \\
\hline & 0,32 & \\
\hline & 0,29 & \\
\hline 90,2 & 0,09 & 0,28 \\
\hline & 0,53 & \\
\hline & 0,40 & \\
\hline & 0,15 & \\
\hline & 0,06 & \\
\hline & 0,05 & \\
\hline 49,3 & 0,02 & 0,20 \\
\hline & 0,05 & \\
\hline 75,5 & 0,73 & \\
\hline
\end{tabular}

(1) $\mathbf{B T}=$ Boituva; $\mathbf{C B}=$ Cabezona; $\mathbf{G N}=$ Guiana; $\mathbf{H T}=$ Huitota; $\mathbf{M I}=$ Manauara Inerme; $\mathbf{M Z}=$ Manzana; $\mathbf{N Q}=$ Natal Queen; PL = Perolera $; \mathbf{P N}=$ Piña Negra $; \mathbf{P R}=$ Pérola $; \mathbf{R D}=$ Rondon $; \mathbf{R I}=$ Roxo Inerme $; \mathbf{R J}=$ Rioja $; \mathbf{R T}=$ Roxo-de-tefé $; \mathbf{S C}=$ Smooth Cayenne; $\mathbf{S R}=$ San Ramón; $\mathbf{T P}=$ Tapiracanga e TV $=$ Turi Verde. $\left({ }^{2}\right) \mathbf{N F P}=\mathrm{n} .{ }^{\circ}$ de flores polinizadas; $\left({ }^{3}\right)$ NTS $=$ $\mathrm{n} .^{\circ}$ total de sementes; $\left({ }^{4}\right) \mathbf{N S V}=\mathrm{n} .{ }^{\circ}$ de sementes viáveis; $\left({ }^{5}\right) \mathbf{P S V}=$ porcentagem de sementes viáveis; $\left({ }^{6}\right) \mathbf{N S V} / \mathbf{F P}=\mathrm{n} .{ }^{\circ}$ de sementes viáveis por flor polinizada. 
segregantes com alta freqüência de genótipos superiores (Loison-Cabot \& Lacoeuilhe, 1990). Vale ressaltar que, em alguns casos, os polinizadores do Perolera (Smooth Cayenne, Manzana, Tapiracanga e Boituva) não se mostraram adequados (de 0,16 a 0,52 semente viável/fruto). Nos outros cultivares comerciais - Pérola e Smooth Cayenne poucos polinizadores dos empregados conseguiram levar à produção de pouco mais de uma semente viável por flor polinizada na fêmea.

Estimou-se, por análise de correlação, a correspondência ou não das médias obtidas para o caráter produção de sementes nas famílias com aquelas conseguidas em polinização aberta, com os mesmos genitores femininos. Obteve-se um coeficiente de correlação $(r=0,82)$, significativo ao nível de $0,07 \%$. Isso indica que os cruzamentos dirigidos realizados foram suficientes para confirmar os dados obtidos em polinização aberta.

Finalmente, no quadro 4, encontram-se os cruzamentos recíprocos realizados, com o intuito de detecção de possíveis efeitos de parentais relacionados à produção de sementes, conforme já obervado para vários caracteres quantitativos, em abacaxi (Loison-Cabot \& Lacoeuilhe, 1990).

Considerando que cada flor de abacaxi possui, no mínimo, um óvulo em condições de ser fertilizado, os resultados mais contrastantes quanto à quantidade de sementes produzidas pode sugerir a ocorrência de incompatibilidade unilateral (sistema gametofítico) do tipo: $9 \mathrm{~S}_{\mathrm{a}} \mathrm{S}_{\mathrm{a}} \times$ o $^{7} \mathrm{~S}_{\mathrm{a}} \mathrm{S}_{\mathrm{b}}$ (e o seu recíproco), onde $S_{a}>S_{b}$. Neste caso, tem-se, de um lado, 50\% pólen $\left(\mathrm{S}_{b}\right)$ com capacidade de germinação no estigma da planta $S_{a} S_{a}$ e, do outro, nenhuma possibilidade de germinação no cruzamento recíproco.

Os cruzamentos envolvendo o cultivar Perolera reforçam essa interpretação porque se descarta a ocorrência de significativa inviabilidade de pólen e óvulo. Assim, os cruzamentos PL x RT, PL x PN e PL x RD, com 4,67, 2,21 e 1,66 sementes por flor polinizada respectivamente, evidenciam que não deve haver problemas nos gametas femininos.
Quadro 4. Comparações entre cruzamentos recíprocos de cultivares e clones de abacaxi quanto ao número de sementes viáveis por flor polinizada

\begin{tabular}{|c|c|c|}
\hline Cruzamento $\left({ }^{l}\right)$ & $\begin{array}{l}\text { N. de flores } \\
\text { polinizadas }\end{array}$ & $\begin{array}{l}\text { N. }{ }^{\circ} \text { de sementes } \\
\text { viáveis/flor } \\
\text { polinizada }\end{array}$ \\
\hline q o & n. ${ }^{\circ}$ & $n .^{\circ}$ \\
\hline$N Q \times R D$ & 27 & 1,52 \\
\hline$R D \times N Q$ & 103 & 0,07 \\
\hline PL $\times \mathrm{RT}$ & 57 & 4,67 \\
\hline RT $\times$ PL & 88 & 0,81 \\
\hline PL $x$ PN & 14 & 2,21 \\
\hline PN $\times$ PL & 9 & 0,67 \\
\hline $\mathrm{PL} \times \mathrm{RD}$ & 98 & 1,66 \\
\hline $\mathrm{RD} \times \mathrm{PL}$ & 113 & 0,46 \\
\hline$B T \times R D$ & 172 & 0,29 \\
\hline $\mathrm{RD} \times \mathrm{BT}$ & 151 & 0,04 \\
\hline $\mathrm{SC} \times \mathrm{PL}$ & 14 & 1,64 \\
\hline $\mathrm{PL} \times \mathrm{SC}$ & 94 & 0,52 \\
\hline PR $\times$ RT & 16 & 0,81 \\
\hline RT $x$ PR & 20 & 0,25 \\
\hline BT X PL & 107 & 0,43 \\
\hline PL $\times$ BT & 159 & 0,16 \\
\hline $\mathrm{TP} \times \mathrm{PL}$ & 74 & 0,53 \\
\hline$P L \times T P$ & 41 & 0,22 \\
\hline $\mathrm{RD} \times \mathrm{GN}$ & 42 & 0,38 \\
\hline GN $x \mathrm{RD}$ & 54 & 0,19 \\
\hline $\mathrm{PL} \times \mathrm{MZ}$ & 16 & 0,25 \\
\hline $\mathrm{MZ} \times \mathrm{PL}$ & 25 & 0,12 \\
\hline$R J \times P N$ & 10 & 3,10 \\
\hline$P N \times R J$ & 11 & 1,91 \\
\hline$R D \times S C$ & 51 & 0,59 \\
\hline$S C \times R D$ & 67 & 0,36 \\
\hline $\mathrm{RT} \times \mathrm{SC}$ & 11 & 0,09 \\
\hline SC $\times \mathrm{RT}$ & 14 & 0,07 \\
\hline $\mathrm{SC} \times \mathrm{TV}$ & 14 & 0,07 \\
\hline TV $\mathrm{x} S \mathrm{C}$ & 61 & 0,02 \\
\hline
\end{tabular}

(1) $\mathbf{A U}=$ Amarelo-de-uaupés; $\mathbf{B T}=$ Boituva; $\mathbf{G N}=$ Guiana; $\mathbf{M Z}$ = Manzana $\mathbf{N Q}=$ Natal Queen $; \mathbf{P L}=$ Perolera $; \mathbf{P N}=$ Piña Negra; $\mathbf{P R}=$ Pérola $; \mathbf{R D}=$ Rondon $; \mathbf{R J}=$ Rioja $; \mathbf{R T}=$ Roxo-de-tefé $;$ $\mathbf{S C}=$ Smooth Cayenne; $\mathbf{T P}=$ Tapiracanga e $\mathbf{T V}=$ Turi Verde. 
Da mesma forma, o cruzamento SC x PL $(1,64$ semente/flor) mostra que o cultivar Perolera, aparentemente, não apresenta pólen com significativo grau de inviabilidade. Em resumo, os dados podem sugerir tratar-se de ocorrência de incompatibilidade unilateral.

As menores produções de sementes foram observadas nos cruzamentos Smooth Cayenne x Turi Verde e Roxo-de-tefé x Smooth Cayenne, devido, provavelmente à presença de incompatibilidade gametofítica (Brewbaker \& Gorrez, 1967).

\section{REFERÊNCIAS BIBLIOGRÁFICAS}

BREWBAKER, J.L. \& GORREZ, D.D. Genetics of selfincompatibility in the Monocot genera, Ananas (pineapple) and Gasteria. American Journal of Botany, Baltimore, 54(5):611-616, 1967.

CABOT, C. Practice of pineapple breeding. Acta Horticulturae, Bangalore, 196:25-36, 1987.
CABRAL, J.R.S.; MATOS, A.P. \& CUNHA, G.A.P. Caracterização morfológico-agronômica de germoplasma de abacaxi. In: CONGRESSO BRASILEIRO DE FRUT.ICULTURA, 9., Campinas, 1987. Anais. Campinas, Sociedade Brasileira de Fruticultura, 1988. v. 1, p. $35-40$.

ESPIRONELO, A. \& TEÓFILO SOBRINHO, J. Florescimento, frutificação e produçăo de mudas de variedades de abacaxizeiros em São Paulo. Revista Brasileira de Fruticultura, Cruz das Almas, 13(2):99-106, 1991.

GiACOMELLI, E.J. \& TEÓfILO SOBRINHO, J. Seleção preliminar de algumas cultivares de abacaxizeiro resistentes à fusariose. In: CONGRESSO BRASILEIRO DE FRUTICULTURA, 7., Florianópolis, 1983. Anais. Florianópolis, Sociedade Brasileira de Fruticultura/EMBRAPA, 1984 . v.1, p.145-161.

LOISON-CABOT, C. \& LACOEUILHE, J.J. A genetic hybridization programme for improving pineapple quality. Acta Horticulturae, Bangalore, 275:395-400, 1990.

SOUTO, F.A. d'A. Variedades e melhoramento do abacaxizeiro. Informe Agropecuário, Belo Horizonte, 7(74):12-14, 1981 .

THOMAS, M.L. \& HILLS, F.J. Agricultural experimentation: design and analysis. New York, John Willey and Sons, 1977. 350p. 